\title{
Anatomía tridimensional y cirugía virtual para procedimientos transobturatrizes
}

\author{
Palma P, Riccetto C, Fraga R, Portugal S, Dambros M, Rincón ME*, Silveira A, Netto NR Jr. \\ Departamento de Urología, Universidad Estadual de Campinas, UNICAMP, Sao Paulo, Brasil. \\ *Instituto Docente de Urologia. Universidad de Carabobo, Valencia, Venezuela.
}

Actas Urol Esp. 2007;31(4):361-365

\section{RESUMEN}

ANATOMÍA TRIDIMENSIONAL Y CIRUGÍA VIRTUAL PARA PROCEDIMIENTOS TRANSOBTURATRIZES

Introducción: La Incontinencia Urinaria de Esfuerzo (IUE) se maneja quirúrgicamente diferente siguiendo la Teoría Integral de la Continencia razón por la cual es importante el conocimiento de las estructuras anatómicas. Desarrollamos un modelo tridimensional basado en la anatomía de la pelvis para el entrenamiento manual de la cirugía y así disminuir la curva de aprendizaje de urólogos y ginecólogos.

Objetivo: Demostrar en modelos sintéticos, las bases anatómicas del manejo transobturatriz en la IUE, cistoceles y en IUE recidivante post tratamiento quirúrgico.

Metodología: El modelo anatómico incluye: huesos pélvicos, las principales capas de los músculos pélvicos, ligamentos y fascias. Los dispositivos quirúrgicos son agujas transobturatrizes en forma de "C" y helicoidales; un sling reajustable de acceso universal y una malla especial autofijable y el sling crossover. Fue realizado un entrenamiento con clases teóricas y prácticas. Las clases prácticas fueron realizadas con las pelvis y los dispositivos de la cirugía transobturatriz. A continuación fueran realizadas cirugias transobturatrizes en ovejas. Al final, se aplicó un cuestionario para verificar el impacto de esta herramienta en la curva de aprendizaje del acceso transobturatriz.

Resultados: Con respecto al concepto anatómico, el 90\% $(n=72)$ de los participantes, clasificó el modelo como muy bueno y $10 \%(n=8)$ como bueno. Como herramienta para el aprendizaje de los procedimientos transobturatrizes, el $100 \%$ aprobó tanto el material escrito como el modelo anatómico tridimensional.

Conclusiones: Este modelo sintético permite entender y poner en práctica las diversas técnicas quirúrgicas, substituyendo los modelos animales, las reconstrucciones tridimensionales computarizadas y las imágenes bidimensionales, contribuyendo así de forma significativa en la curva de aprendizaje.

Palabras claves: Incontinencia urinaria de esfuerzo. Piso pélvico. Anatomía tridimensional. Corrección quirúrgica de incontinencia.

\section{ABSTRACT}

\section{THREE DIMENSIONAL ANATOMY AND VIRTUAL SURGERY FOR TRANSOBTURATOR APPROACH}

Introduction: Stress Urinary Incontinence (SUI) may be managed by transobturator approach. We developed a threedimensional model, for understanding the surgical anatomy and manual training as well, in order to reduce the learning curve for pelvic surgeries.

Objective: To demonstrate in synthetic models, the anatomical basis for the management of SUI and cystocele.

Method: The anatomical model includes: pelvic bones, the main layers of the pelvic muscles, ligaments and fascias. The surgical devices were transobturator needles, synthetic slings and meshes for anterior vaginal wall repair. The workshop was carried out with an anatomical overview and hands-on training in this tridimentional models and finally surgery in animals. At the end, a questionnaire was applied to verify the impact of this tool in the learning process and trainee satisfaction.

Results: As far as the anatomical concept, 90\% $(\mathrm{n}=72)$ of the participants classified this model as very good and $10 \%$ $(\mathrm{n}=8)$ as good. As a tool for understanding the tridimentional pelvic floor anatomy applied to transobturator procedures there were $100 \%$ of approval.

Conclusions: This synthetic model allows for understanding the pelvic floor tridimentional anatomy and surgical procedures as well. Further skill is got in the animal model reducing the learning curve for transobturator procedures. 
$\mathrm{L}$ a Incontinencia Urinaria de Esfuerzo (IUE) definida por la Sociedad Internacional de Continencia (ICS) como la "pérdida involuntaria de orina desencadenada con el esfuerzo físico"1, tiene actualmente un manejo quirúrgico diferente siguiendo los nuevos paradigmas de la Teoría Integral de la Continencia concebida por Petros, quien explica de forma conjunta los mecanismos fisiopatológicos responsables de la misma así como los sintomas habitualmente coexistentes, considerando que la IUE ocurre por alteraciones de los tejidos de soporte suburetral, ligamentos y músculos del piso pélvico ${ }^{2}$. Por esta razón es importante el conocimiento de las estructuras anatómicas y la correcta interpretación de los mecanismos envueltos en la pérdida de orina. La anatomía pélvica puede ser definida como el conjunto de huesos, músculos, ligamentos y órganos que contribuyen para la función normal del piso pélvico ${ }^{3}$.

Los ligamentos, músculos y fascias constituyen un sistema músculo-elástico que ofrece forma y función a los órganos pélvicos. Fascia es definida como el tejido fibromuscular responsable de suspender y dar estabilidad a los órganos o unirlos a los músculos. Los espesamientos de éstas son denominados ligamentos, y su función es ofrecer puntos de suspensión y anclaje de los músculos. Los principales ligamentos del piso pélvico son: Ligamento Uretral Externo (LUE), que está localizado anteriormente a la membrana perineal; Ligamento Pubo-Uretral (LPU), que se localiza arriba de la membrana perineal; Arco Tendíneo de la Fascia Pélvica (ATFP); Ligamento Cardinal y Útero sacro. Los músculos del piso pélvico forman tres capas: superior, media, e inferior (Petros 1997). La capa superior está formada por la porción anterior del músculo pubo-coccígeo anteriormente y elevador del ano posteriormente, la capa media formada por el músculo longitudinal del ano y la capa inferior por los músculos localizados junto a la membrana perineal, y esfinter anal ${ }^{3}$.

$\mathrm{El}$ concepto de apoyo posterior de la uretra sin tensión fue introducido por McGuire, pero la corrección era en el cuello vesical, lo cual ocasionó un gran número de disfunciones miccionales y enteroceles ${ }^{4}$.

Basándose en el defecto de soporte suburetral (hamaca), se introdujo el swing libre de tensión a nivel de la uretra media. En esta teoría, el para- digma de corrección del defecto es el mantenimiento del eje vaginal sin elevación o angulación de la uretra. Como el soporte suburetral depende de la acción conjunta de los ligamentos pubouretrales, uretropélvicos y tejido suburetral, se consideró que la corrección debería ser conjunta ${ }^{5}$. Entonces, la Teoría Integral simplificó la técnica, abogando por la creación de neo-ligamentos con mallas sintéticas, e introdujo el concepto de sling autofijable, y además lo colocó a nivel de la uretra media, lo cual contribuyó significativamente para disminuir la retención urinaria en el postoperatorio $^{4}$.

Delorme, en el 2001, fue el primero en reportar el acceso transobturatriz utilizando un abordaje minimamente invasivo y siguiendo los principios de la biocirugía 5 .

Se han descrito buenos resultados alrededor del mundo con el empleo del sling transobturatriz. En un estudio con 117 pacientes operadas en el Hospital de Zone, Suiza, se alcanzó un 92\% de cura con $5 \%$ de lesiones menores ${ }^{6}$. La experiencia en North Queensland fue de $81,3 \%$ de cura evidenciada mediante reevaluación urodinámica ${ }^{7}$. Dávila et al describieron $89,4 \%$ de continencia total o parcial con un seguimiento postoperatorio de 14 semanas, en un estudio que incluyó 169 pacientes con IUE, no presentándose ninguna lesión vesical ni intestinal, y con 3,4\% de retención postoperatoria ${ }^{8}$. Sin embargo, hay autores que describen lesiones menores como el hematoma retropúbico producto de lesión vascular', y lesiones mayores con sangrado excesivo que ameritaron transfusión, así como neuropatía del obturador en el postoperatorio, infecciones que ameritaron tratamiento antibiótico endovenoso y hasta retiro del sling ${ }^{10}$, y perforación vesicall $^{10-12}$.

Nosotros desarrollamos un modelo tridimensional basado en la anatomía de la pelvis femenina para el entrenamiento manual de la cirugía y de esta forma, disminuir la curva de aprendizaje de urólogos y ginecólogos.

\section{OBJETIVO}

Demostrar en modelos sintéticos, las bases anatómicas del manejo transobturatriz en la IUE, cistoceles y en IUE recidivante post tratamiento quirúrgico. 


\section{MÉTODOS}

Desarrollamos un modelo tridimensional del piso pélvico femenino y agujero obturador. El modelo anatómico incluye: huesos pélvicos, las principales capas de los músculos pélvicos, ligamentos y fascias. La pelvis es hecha de resina con moldes confeccionados a partir de pelvis humanas. Los músculos fueron confeccionados con goma flexible, siendo fijados en la pelvis con velcro. Fascias, ligamentos y membranas fueron confeccionadas con malla de polipropileno. Los dispositivos quirúrgicos son agujas transobturatrizes en forma de "C" y otras helicoidales; un sling reajustable de acceso universal y una malla especial autofijable para la reparación de prolapsos central y laterales de la pared vaginal anterior (NAZCA), y el sling crossover.

$\mathrm{El}$ entrenamiento fue realizado en 1 día con módulos de clases teóricas y clases prácticas. Las clases teóricas fueron apoyadas con material escrito que contenía la descripción anatómica del piso pélvico, métodos de evaluación de los prolapsos y técnicas quirúrgicas. Las clases prácticas fueron realizadas con las pelvis, $\mathrm{y}$ los participantes fueron divididos en grupos de 10. Cada participante tuvo oportunidad de armar el modelo pélvico por lo menos $1 \mathrm{vez}$, y realizar las cirugias.

Al final, se aplicó un cuestionario para verificar el impacto de esta herramienta en la curva de aprendizaje del acceso transobturatriz. El cuestionario consistió en 3 preguntas:
1) ¿Cuál es su evaluación del modelo anatómico como herramienta de aprendizaje de la anatomía pélvica?

2) ¿Cuál es su evaluación del modelo anatómico como herramienta de aprendizaje de procedimientos por vía transobturatriz?

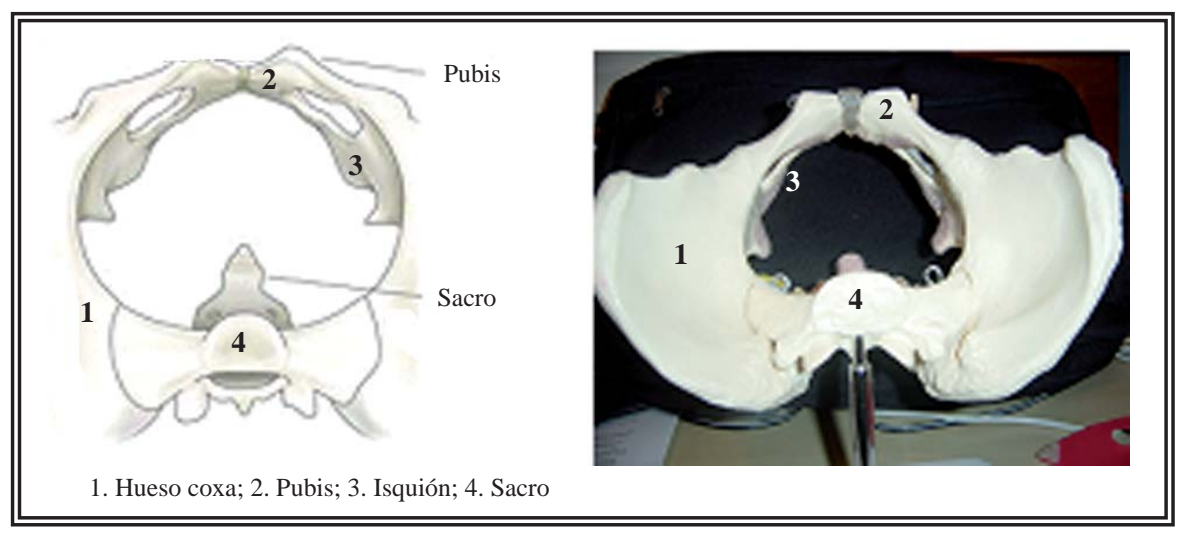

FIGURA 1. Pelvis ósea - Vista ósea.

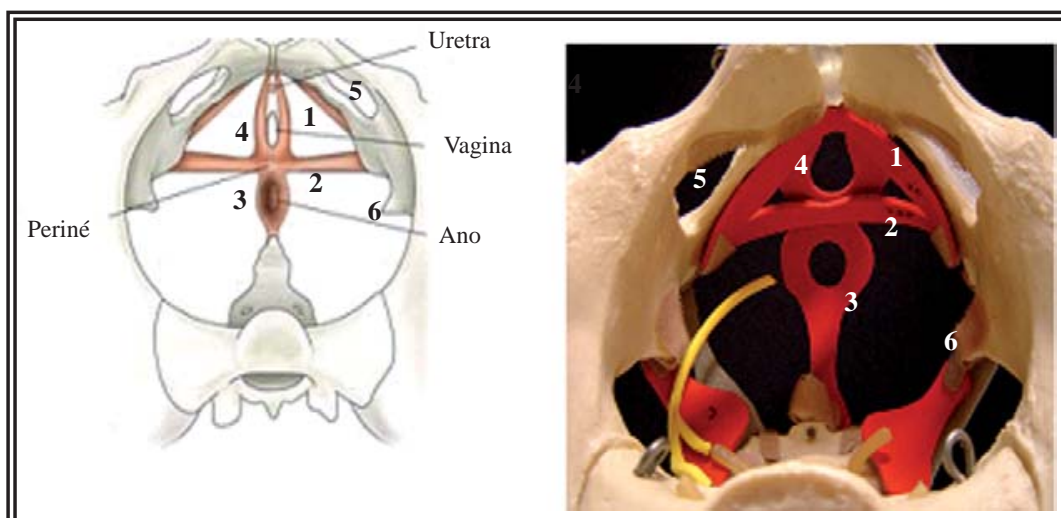

1. Músculo isquiocavernoso; 2. M. transverso superficial del periné; 3. M. Esfínter anal externo; 4. M. bulbocavernoso; 5. Agujero obturador; 6. Espina isquiática.

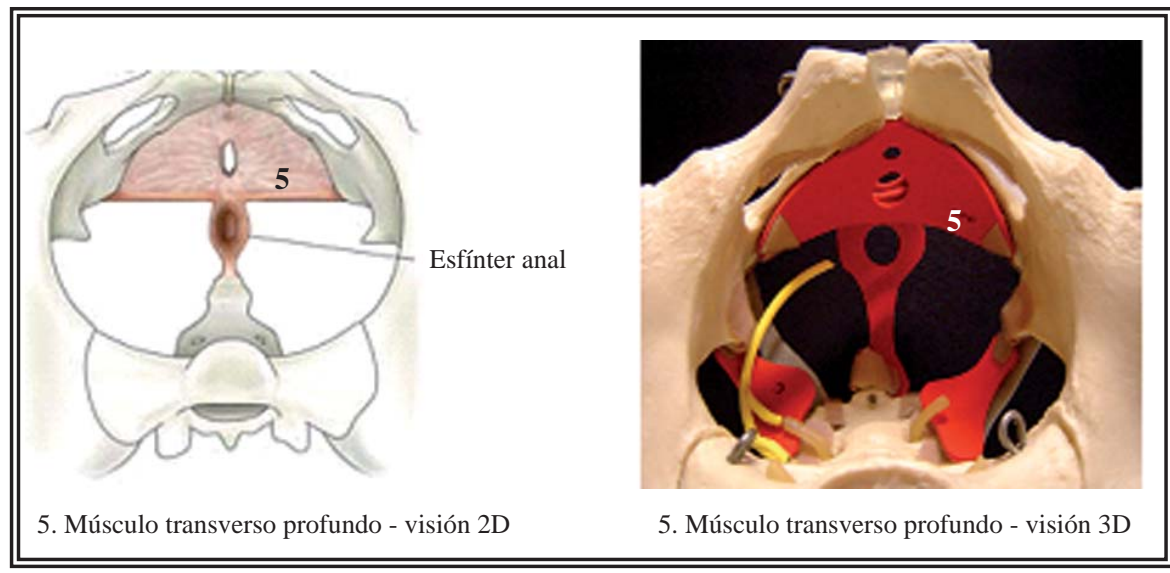

FIGURA 3. Visión superior de la cavidad pélvica. 
3) ¿Cuál es su evaluación del material didáctico escrito como herramienta de aprendizaje?

Las respuestas fueron graduadas en: a.-muy bueno, b.bueno, c.-regular, d.-malo, e.muy malo. Hubo 80 cirujanos (urólogos y ginecólogos) participando en el entrenamiento manual, ninguno miembro del grupo instructor.

\section{RESULTADOS}

La confección de los moldes fue realizada a partir de figuras bidimensionales del piso pélvico femenino presentes en la literatura.

Debajo hacemos una comparación de las formas bidimensional y tridimensional. Están representadas estructuras del piso pélvico en tamaño real, siguiendo las referencias anatómicas y que pueden ser colocadas en posición para entrenamiento quirúrgico (Figs. 1, 2, 3, 4, 5, 6 у 7).

En relación al material didáctico escrito, el 85\% $(n=68)$ de los participantes, lo clasificó como muy bueno. Con respecto al concepto anatómico, el 90\% (n=72) de los participantes, clasificó el modelo como muy bueno y $10 \%(n=8)$ como bueno. Como herramienta para el aprendizaje de los procedimientos transobturatrizes, el 100\% aprobó tanto el material escrito como el modelo anatómico tridimensional.

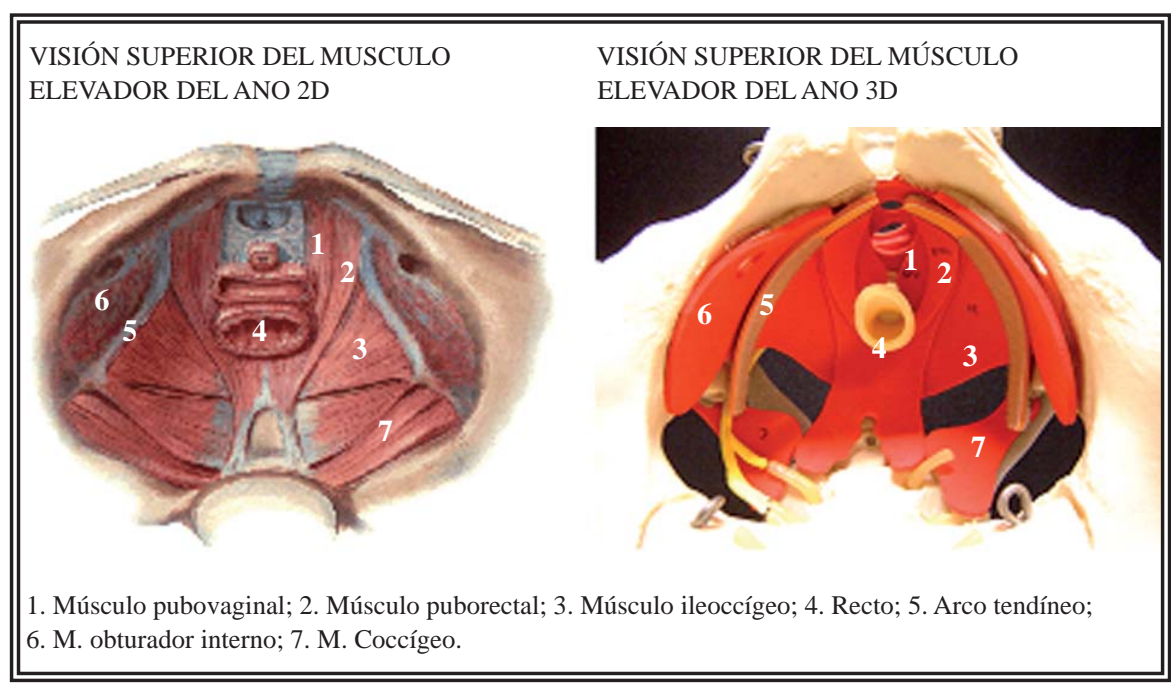

FIGURA 4. Visión superior de la cavidad pélvica.

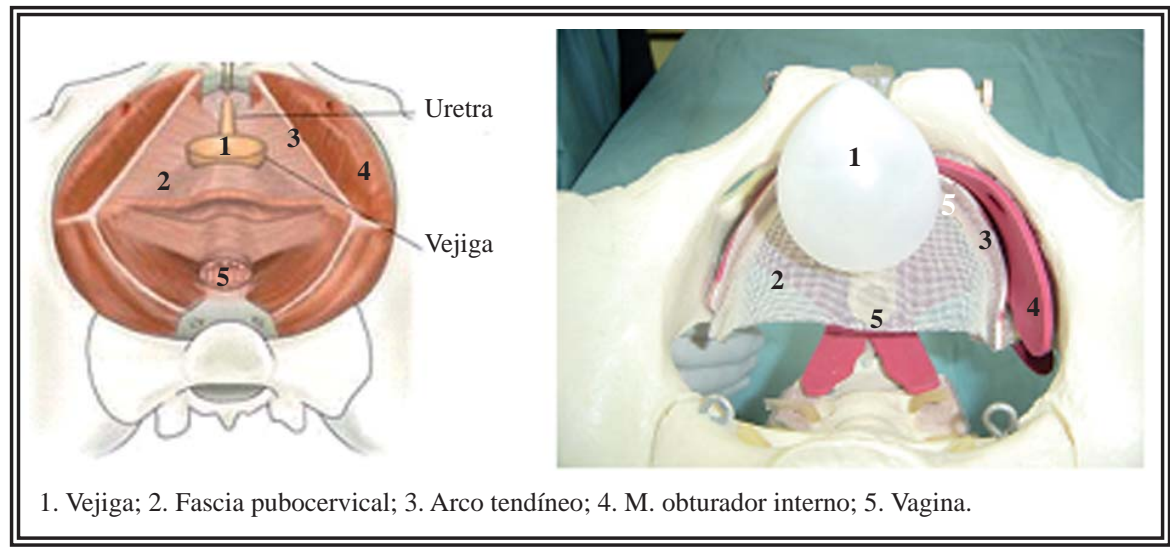

FIGURA 5. Fascia pubocervical y sus relaciones anatómicas.

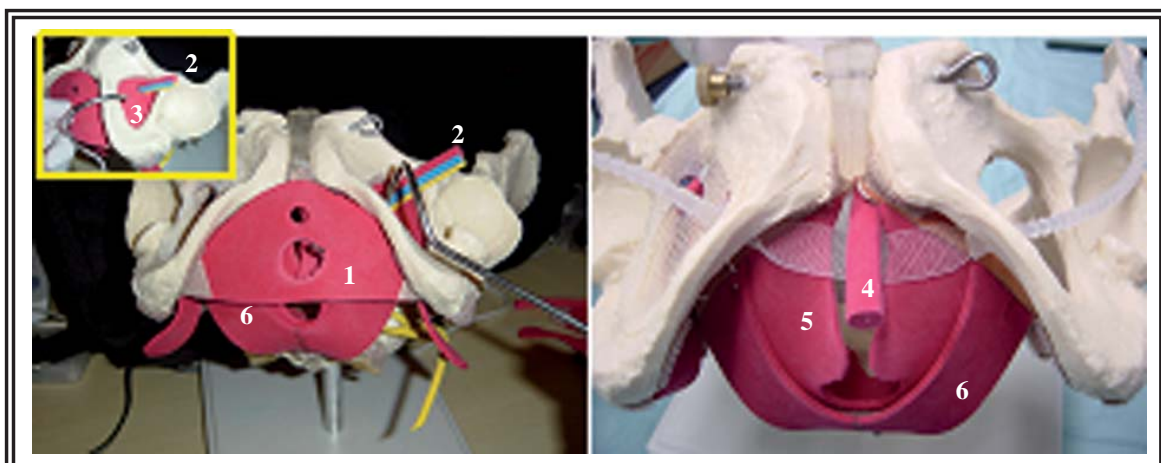

1. Músculo transverso del periné; 2. Conjunto vásculo-nervioso del canal obturador; 3. Músculo obturador interno; 4. Uretra; 5. Músculo puborectal; 6. Músculo ileococcígeo; 7. Membrana obturadora.

FIGURA 6. Técnica quirúrgica transobturadora.

\section{CONCLUSIONES}

La técnica quirúrgica empleada para la corrección de la IUE en la actualidad ofrece menores riesgos de complicaciones para la paciente pero aún no está exenta de ellos, por eso es fundamental el conocimiento de la anatomía pélvica. Este modelo sintético de tamaño real permite entenderla y poner en práctica las diversas técnicas 


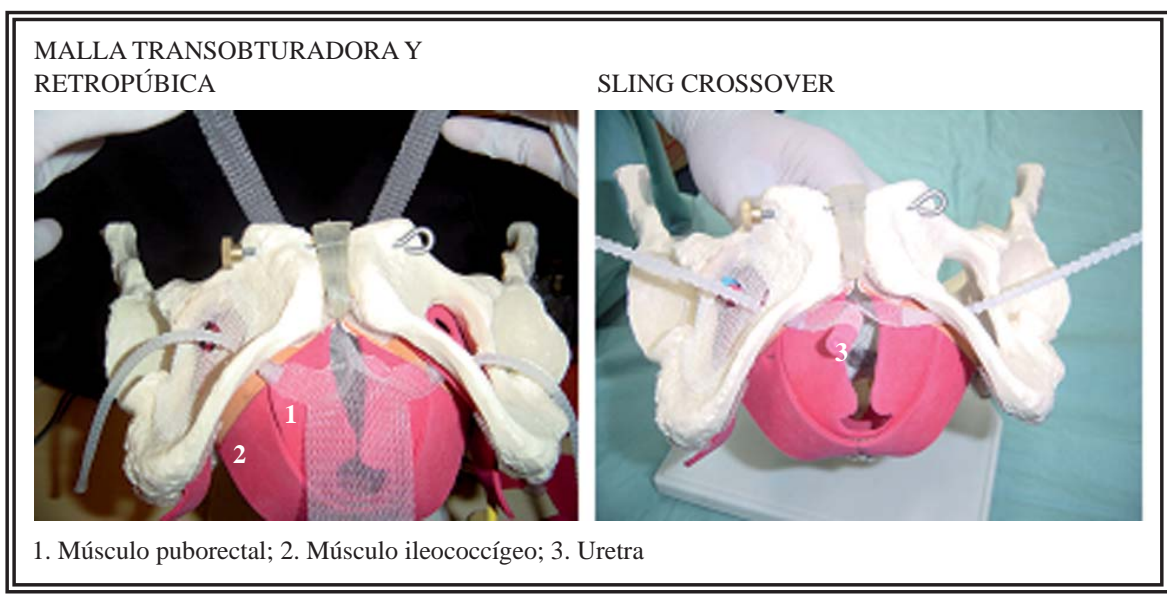

FIGURA 7. Técnica quirúrgica transobturadora.

Fuente de Figuras 1 a 7: Palma Paulo, Riccetto Cássio. Anatomía Tridimensional Aplicada ao Tratamento Cirúrgico das Disfunçoes do Assoalho pélvico Feminino. Campinas, SP: Sociedade Brasileira de Urologia, 2005; pp 8-12.

quirúrgicas, substituyendo los modelos animales, las reconstrucciones tridimensionales computarizadas y las imágenes bidimensionales, contribuyendo así de forma significativa en una reducción de la curva de aprendizaje.

\section{REFERENCIAS}

1. The International Continence Committee of Standarisation of Terminolgy. The Standarisation of terminology of the lower urinary tract function. Seventh Report Neurourol Urodynam 988.

2. Petros PE and Ulmsten U. An Integral Theory of Female Urinary Incontinence. -Acta Obstet Gynecol Scand Suppl. 1990;153:7-31.

3. Palma Paulo, Riccetto Cássio. Anatomia Tridimensional Aplicada ao Tratamento Cirúrgico das Disfunções do Assoalho Pélvico Feminino. Campinas,SP: Sociedade Brasileira de Urologia, 2005; pp 8-12.

4. Palma Paulo. Slings: Por que tantos?. In Paulo Palma, Nelson Rodrigues Netto Jr. Catium: Curso Avançado da Incontinência Urinária na Mulher. São Paulo: Legnar Informática \& Editora Ltda., agosto 2005;pp 89. 106(5):1199-1202.
5. Riccetto Cássio, Petros Peter. Aplicações Clínicas da Teoria integral da Continência. In Paulo Palma, Nelson Rodrigues Netto Jr. Catium: Curso Avançado da Incontinência Urinária na Mulher. São Paulo: Legnar Informática \& Editora Ltda., agosto 2005;pp 24.

6. Spinosa JP, Dubuis PY: Suburethral sling inserted by the transobturator route in the treatment of female stress urinary incontinence: Preliminary results in 117 cases. 2005; 123(2):212-217.

7. Naidu A, Lim YN, Barry C, Goodwin S, Corstiaans A, Rane A: Transobturator tape for stress incontinence: the North Queensland experience. Aust NZJ Obstet Gynaecol. 2005 Oct;45(5):446-449.

8. Davila, G W. Johnson, J D. Serels, S. Early US Experience with a Transobturator Sling to Treat Stress Incontinence. Int. Urogynecol J Pelvic Floor Dysfunct. 2005 Nov-Dec: 16(6):487-491.

9. Rajan S, Kohli N. Retropubic Hematoma After Transobturator Sling Procedure. Obstet Gynecol.2005 Nov;

10. Hamilton Boyles S, Gregory WT, Clark A, Edwards S. Complications associated with trans-obturator sling procedures. Conferencia ICS 2005.

11. Minaglia S, Ozel B, Klutke C, Ballard C, Klutke J: Bladder injury during transobturator sling. Urology. 2004 Aug; 64(2):376-377.

12. Hermieu JF, Messas A, Delmas V, Ravery V, Dumonceau O, Boccon-Gibod L. Bladder injury after TVT transobturator. Prog Urol 2003 Feb;13(1):115-117.

Correspondencia autor: Dr. P. Palma

Departamento de Urología.

Universidad Estadual de Campinas

UNICAMP, Sao Paulo. Brasil

E-mail autor: ppalma@uol.com.br

Información artículo: Original - Incontinencia urinaria esfuerzo

Trabajo recibido: enero 2007

Trabajo aceptado: marzo 2007 